\title{
Orientational Ordering in Spatially Disordered Dipolar Systems
}

\author{
G. Ayton*, M. J. P. Gingras ${ }^{\dagger}$, and G. N. Patey* \\ * Department of Chemistry, University of British Columbia, Vancouver, British Columbia, Canada V6T $1 Z 1$ \\ † TRIUMF, 4004 Wesbrook Mall, Vancouver, British Columbia, Canada V6T 2A3
}

(November 5, 2018)

This letter addresses basic questions concerning ferroelectric order in positionally disordered dipolar materials. Three models distinguished by dipole vectors which have one, two or three components are studied by computer simulation. Randomly frozen and dynamically disordered media are considered. It is shown that ferroelectric order is possible in spatially random systems, but that its existence is very sensitive to the dipole vector dimensionality and the motion of the medium. A physical analysis of our results provides significant insight into the nature of ferroelectric transitions.

PACS numbers: 64.70.Md, 77.80.-e, 82.20Wt

Recently, spatially disordered dipolar materials have attracted considerable attention. Diluted lattices 值3, fluid phases [4.5] and amorphous frozen ferrofluids |6] have been examined experimentally [1],2,6], theoretically [3,711] and with computer simulations [3]5]. It has been shown that in the absence of long-range positional order, the strong spatial-orientational coupling intrinsic to dipolar forces can lead to interesting new phase behavior. For example, frozen ferrofluids containing magnetic particles in a non-magnetic solvent exhibit magnetic irreversibilities reminiscent of randomly frustrated magnetic spin glasses [12]. On the other hand, computer simulations of simple dipolar fluids clearly indicate the existence of a ferroelectric liquid crystal phase 4,5$]$.

A simple interpretation of these observations might be as follows. In frozen ferrofluids, the quenched positional disorder creates random frustration and the system behaves as a spin glass [12]. The fluid systems [4,5] differ from the frozen case in that the strongly coupled translational and rotational degrees of freedom are in full thermal equilibrium. This allows the development of shortrange spatial correlations resembling those seen in the ferroelectric tetragonal-I lattice [13] and, consequently, ferroelectric order develops in the liquid phase [it. In view of these observations, and recalling that perfect crystals exhibit ferroelectric or antiferroelectric long-range order depending on the lattice symmetry [13,14], one might argue that specific spatial correlations are required for ferroelectric order.

In recent papers Zhang and Widom 11] have put forward a mean field theory for spatially disordered dipolar systems. They argue that the long-range nature of the dipolar forces plays a key role in yielding ferroelectric order, and that this is not explicitly included in the sim- ple interpretation given above. More importantly, Zhang and Widom propose that, despite the strong frustration present in randomly frozen systems, long-range ferroelectric order is possible above a critical density. Their work implies that the spin-glass behavior observed in ferrofluids [6] results from the low concentration of magnetic particles, whereas the ferroelectric liquid crystalline phase found in computer simulations of dipolar fluids [4.5] arises because of the high particle density considered. In the present letter we examine the validity of this argument and address the general question "Can long-range ferroelectric order spontaneously arise in a system without fined-tuned positional correlations?"

We investigate the behavior of dense spatially disordered dipolar systems using constant temperature molecular dynamics (MD) and Monte Carlo (MC) simulations. Systems where the dipole vector has one, two and three components are considered. The first two of these are commonly referred to as the Ising and XY models and for notational simplicity we shall refer to the three component dipole as the XYZ model. In all cases the pair potential, $u(12)$, is of the generic form

$$
u(12)=4 \varepsilon(\sigma / r)^{12}+u_{D D}(12),
$$

where $4 \varepsilon(\sigma / r)^{12}$ and

$$
u_{D D}(12)=-3\left(\boldsymbol{\mu}_{1} \cdot \mathbf{r}\right)\left(\boldsymbol{\mu}_{2} \cdot \mathbf{r}\right) / r^{5}+\boldsymbol{\mu}_{1} \cdot \boldsymbol{\mu}_{2} / r^{3}
$$

are the soft-sphere and dipole-dipole interactions. The parameters $\varepsilon$ and $\sigma$ are the fundamental units of energy and length, $\boldsymbol{\mu}_{i}$ is the dipole of particle $i$ and $\mathbf{r}$ is the intermolecular vector. The long-range dipolar interactions were taken into account using periodic boundary conditions and the Ewald summation method assuming a perfectly conducting surrounding continuum $4,15,17$. The existence of a ferroelectric phase can be detected by calculating the average polarization $P$ per particle defined as $P=1 / N\left\langle\left|\sum_{i=1}^{N} \hat{\boldsymbol{\mu}}_{i} \cdot \hat{\mathbf{d}}\right|\right\rangle$, where $\hat{\mathbf{d}}$ is a unit vector in the direction of the total instantaneous moment, $\mathbf{M}=\sum_{i=1}^{N} \boldsymbol{\mu}_{i}$, and $N$ is the number of particles in the system.

It is convenient to characterize dipolar soft-sphere systems by the reduced density, $\rho^{*}=N \sigma^{3} / V$, the reduced temperature, $T^{*}=k_{\mathrm{B}} T / \varepsilon$, and the reduced dipole moment, $\mu^{*}=\left(\mu^{2} / \varepsilon \sigma^{3}\right)^{1 / 2}$, where $V$ is the volume of the sample, $T$ is the absolute temperature and $k_{\mathrm{B}}$ is the Boltzmann constant. All results explicitly presented are for $\mu^{*}=4$ and $\rho^{*}=0.8$. This density is well within the 
range for which Zhang and Widom predict a ferroelectric phase 18]. For $\mu^{*}=4$ and $\rho^{*}=0.8$, Zhang and Widom predict a ferroelectric phase for the Ising case if $T^{*} \leq 35.2$ and for the XYZ model if $T^{*} \leq 4.8$.

We first consider frozen systems. Suitable spatially disordered configurations were generated by carrying out an MD simulation of a soft-sphere fluid at $T^{*}=10.5$ and $\rho^{*}=0.8$. Fluid-like configurations were then selected at random for dipolar rotational MD simulations. Following this approach we could obtain a frozen state at a much higher density than is possible from a random parking algorithm. Unfortunately, it is impossible to have a truly "random" and structureless spatial configuration (i.e., with the radial distribution function $g(r)$ equal to one for $r \geq \sigma$ [1]) at this density. However, at $T^{*}=10.5$ the local structure in the soft-sphere fluid is weak and very short-ranged.

Polarization results for randomly frozen systems are shown in Fig. 1 19]. The XY and XYZ values were obtained with MD simulations. The discrete nature of the Ising model renders it inappropriate for MD so MC calculations were used. The average polarization obtained at the lowest temperature where equilibrium could be achieved, $T_{\min }^{*}$, is plotted vs $1 / N$. The values of $T_{\min }^{*}$ are 10.0, 4.0, and 3.5 for the Ising, XY and XYZ models, respectively. Below these temperatures $\mathrm{MD}$ or $\mathrm{MC}$ runs for the same configuration started from perfectly ordered and disordered states (i.e., two replicas) did not converge to the same result within a reasonable computation time (i.e., about a week). Possibly with greater computational effort $T_{\text {min }}^{*}$ could be pushed a little lower, but the values given above are well within the range where Zhang and Widom predict a ferroelectric phase. For the Ising model the equilibrated system at $T^{*}=10.0$ is nearly $100 \%$ polarized. Moreover, for the Ising case the polarization at $T_{\min }^{*}$ exhibits very little number dependence and certainly does not appear to vanish in the thermodynamic limit. This, together with the plot of $P$ vs $T^{*}$ and heat capacity calculations (see Fig. 3 below), strongly suggests that ferroelectric order develops spontaneously in the spatially disordered Ising system with the transition occurring at $T^{*} \approx 25$ for $\rho^{*}=0.8$. We note that this transition temperature is much lower than the value $\left(T^{*}=35.2\right)$ predicted by Zhang and Widom.

The situation for the XY and XYZ models is very different. Although significant polarization was observed at finite temperatures, $P$ decreases monotonously with increasing system size and appears to approach zero for an infinite system. Furthermore, the behavior of various spin-glass correlation functions and susceptibilities 12 suggests that both systems are entering an anisotropic spin-glass phase at nonzero temperature 20], and that the observed polarization for the XY and XYZ models is due to a combination of short-range ferroelectric correlations and finite-size effects. Test calculations for the XYZ model using a denser frozen soft-sphere configura- tion (i.e., $\rho^{*}=1.0518, T^{*}=10.5$ ) also showed no long-range ferroelectric order. In brief, for the randomly frozen XY and XYZ models we find no evidence of a ferroelectric state in the thermodynamic limit. This clearly disagrees with the calculations of Zhang and Widom which for the XYZ model predict a stable ferroelectric phase well within the temperature-density range considered here.

In order to gain further insight into the nature of ferroelectric order (or the lack thereof) in spatially random systems, we consider a "toy model" where the translational motion is completely decoupled from the dipolar interactions. The soft-sphere fluid acts as a "substrate" which moves at a fixed translational temperature independent of the embedded dipoles. The dipoles themselves interact and are equilibrated at a different rotational temperature. Of course, the "equilibrium" state achieved by the dipoles will depend on the underlying motion of the substrate. This model is similar in spirit to those used in recent studies of non-equilibrium phase transitions in magnetic systems subject to Levy flights [21. It must be emphasized that this technique is presented only as a useful simulation tool and we do not imply any real physical mechanism for the decoupling. The moving substrate is a means of simulating dipolar systems in a dynamically random medium that lacks any specific spatial correlations which may favor ferroelectric ordering. The translational diffusion rate of the substrate can be controlled by the particle mass. Extrapolation to infinite mass should provide information about the randomly frozen system.

In Fig. 2, we have plotted $P$ vs $T^{*}$ (rotational) for the XYZ model. Here, the decoupled substrate is a softsphere fluid again at $\rho^{*}=0.8$ and $T^{*}($ translational $)=$ 10.5. It is convenient to introduce the reduced mass $m^{*}=$ $m / m^{\prime}$, where $m^{\prime}$ is a reference mass defined such that the reduced simulation timestep $\Delta t^{*} \equiv\left(\epsilon / m^{\prime} \sigma^{2}\right)^{1 / 2} \Delta t=$ 0.00125 . Figure 2 includes results for $m^{*}=1,5$ and 10 . Spontaneous polarization develops for all systems and the temperature at which $P$ begins to grow decreases with increasing mass. For example, from the $P$ vs $T^{*}$ plot there appears to be a ferroelectric transition at $T^{*} \approx 2$ for $m^{*}=1$. To verify that this is a real thermodynamic transition, we have calculated the Binder ratio [12], $g_{\text {Binder }} \equiv(5 / 2)-(3 / 2)\left\langle|\mathbf{M}|^{4}\right\rangle /\left\langle|\mathbf{M}|^{2}\right\rangle^{2}$, for systems with 64,108 and 256 particles. A plot of $g_{\text {Binder }}$ vs $T^{*}$ (see Fig. 2, inset) shows a clear crossing, and hence a transition, at $T^{*}=1.9$. Simulations were also carried out with $m^{*}=20$ but no significant orientational order was found above $T^{*}=0.1$. Very slow convergence prevented calculations at lower temperatures.

We have also investigated dynamically disordered XY and Ising systems. The XY model behaves much like the XYZ system described above. For the Ising case, rotational dynamics cannot be used and a suitable Monte Carlo scheme which allowed the substrate to move independent of the Ising dipoles was employed. $P$ vs $T^{*}$ 
results for $m^{*}=1, m^{*}=5$, and the randomly frozen system $\left(m^{*}=\infty\right)$ are shown in Fig. 3. We see that for the Ising model the ordering behavior is essentially independent of mass and that the results for the dynamically disordered system with $m^{*}=5$ lie very close the those for the randomly frozen case. Heat capacities obtained by differentiating the average dipolar energy with respect to the rotational temperature are also shown in Fig. 3. The randomly frozen and $m^{*}=5$ results are very similar and indicate a phase transition at $T^{*} \approx 25$.

The dependence of the ferroelectric transition temperature on particle mass is shown in Fig. 4. The transition temperatures were estimated from the heat capacities and results are included for the XY and XYZ models. Results for the Ising system are not plotted because the transition temperature is essentially independent of the mass. We see that as the mass increases the transition temperature drops for both the XY and XYZ models. As noted earlier, for large masses and low temperatures convergence becomes prohibitively slow, but it seems reasonable to assume that the graph would simply continue with the transition temperature approaching zero in the infinite mass limit. This is consistent with the fact that we did not find a ferroelectric phase for randomly frozen systems at finite temperatures.

The present and previous results can be interpreted as follows. It is useful to divide the local field, $\mathbf{E}_{\text {local }}$, experienced by a particle in an infinite medium into two parts such that, $\mathbf{E}_{\text {local }}=\mathbf{R}+\mathbf{E}$, where $\mathbf{R}$ is a reaction field contribution 9, 15 and $\mathbf{E}$ is everything else. The reaction field arises from the long-range nature of the dipolar interactions; it is independent of the spatial correlations and favors ferroelectric order. The remaining contribution, $\mathbf{E}$, is sensitive to positional correlations and may or may not favor ferroelectric order. Thus if $\mathbf{R}$ dominates a ferroelectric phase is to be expected, but if $\mathbf{E}$ is important the existence or nonexistence of a ferroelectric phase will depend on the details of the spatial correlations. This simple picture allows us to rationalize the various systems considered. For fully coupled dipolar fluids [4,5] the short-range spatial correlations (and hence E) can adjust (i.e., become tetragonal-I-like) in order to favor (or at least allow) a ferroelectric state. On the other hand, for randomly frozen systems the spatial correlations cannot adjust and at equilibrium $\mathbf{E}$ dominates $\mathbf{R}$ frustrating the formation of a ferroelectric phase except for the Ising case. Apparently, the discrete nature of the Ising model makes it much less susceptible to the development of disordering fields than are the $\mathrm{XY}$ and XYZ systems. The reaction field dominates in the Ising system and gives rise to a ferroelectric state.

The behavior of the dynamically disordered systems can also be understood. If the translational diffusion of the substrate is sufficiently fast compared to the dipolar reorientational time, the extent of spatially dependent frustrating correlations is limited, $\mathbf{R}$ can prevail over $\mathbf{E}$, and a ferroelectric phase is possible. As the mass of a substrate particle is decreased, the translational motion becomes faster and the above condition is met at higher and higher rotational temperatures. Thus the observed transition temperatures increase with decreasing mass. As the substrate particles become sufficiently light, the transition temperature is determined only by the reaction field and hence becomes independent of mass. In fact, $m^{*}=1$ gives essentially this limiting behavior and reducing the mass further has little effect on the transition temperature.

In conclusion, the answer to the question raised at the outset is a subtle one. Our results for the frozen Ising system and for the dynamically disordered XY and XYZ models clearly demonstrate that it is possible to have ferroelectric states without fine-tuned positional correlations. However, they also show that if a ferroelectric phase is to exist in a positionally random system, the long-range spatially-independent correlations arising through the reaction field must dominate the shorterranged position-sensitive correlations which generally act to frustrate ferroelectric order. For the Ising system the discrete nature of the interaction (i.e., there is just not much opportunity for favorable interactions among neighbors) limits the build up of short-range disordering correlations and there is a clear ferroelectric transition. For the dynamically random systems the orientational correlations opposing ferroelectric order are reduced by the motion of the substrate resulting in a ferroelectric phase. On the other hand, for the randomly frozen XY and XYZ models we find no indication of a ferroelectric phase at finite temperature. Rather, the disordering fields dominate and these systems appear to form nonferroelectric spin glasses at low temperature. Evidence for this is provided both by our direct simulations of frozen systems and by the extrapolation of the dynamically disordered results to infinite mass. This conclusion must remain a little tentative because direct MD simulations at very low temperatures are not practical. Nevertheless, a ferroelectric phase in the randomly frozen XY and XYZ models seems highly unlikely.

\section{ACKNOWLEDGMENTS}

We thank Z. Rácz, M. Widom and H. Zhang for useful discussions. The financial support of the National Science and Engineering Research Council of Canada is gratefully acknowledged. 
[1] B.E. Vugmeister and M.D. Glinchuk, Rev. Mod. Phys. 62, 4 (1990).

[2] U.T. Höchli and M. Maglione, J. Phys. Condens. Matter 1, 2241 (1989).

[3] H-J Xu, B. Bergersen, and Z. Racz, J. Phys. Condens. Matter 4, 2035 (1992); H-J Xu, Ph.D. thesis, University of British Columbia (1992).

[4] D. Wei and G.N. Patey, Phys. Rev. Lett. 68, 2043 (1992); Phys. Rev. A. 46, 7783 (1992).

[5] J.J. Weis, D. Levesque, and G.J. Zarragoicoechea, Phys. Rev. Lett. 69, 913 (1992).

[6] W. Luo, S.R. Nagel, T.F. Rosenbaum, and R. E. Rosenweig, Phys. Rev. Lett. 67, 2721 (1991).

[7] M.A. Zaluska-Kotur and M. Cieplak, Europhys. Lett. 23, 85 (1993).

[8] B. Mertens, K. Levin, and G.S. Grest, Phys. Rev. B 49, 15374 (1994).

[9] D. Wei, G.N. Patey, and A. Perera, Phys. Rev. E 47, 506 (1993).

[10] B. Groh and S. Dietrich, Phys. Rev. E 50, 3814 (1994); Phys. Rev. Lett. 72, 2422 (1994).

[11] H. Zhang and M. Widom, J. Magn. Magn. Mater. 122, 119 (1993); Phys. Rev. B 51, 8951 (1995).

[12] K. Binder and A.P. Young, Rev. Mod. Phys. 58, 801 (1986); K.H. Fischer and J.A. Hertz, Spin Glasses (Cambridge University Press, Cambridge, 1991).

[13] R. Tao and J.M. Sun, Phys. Rev. Lett. 67, 398 (1991).

[14] J.M. Luttinger and L. Tisza, Phys. Rev. 70, 954 (1946).

[15] S.W. de Leeuw, J.W. Perram, and E.R. Smith, Ann. Rev. Phys. Chem. 37, 245 (1986); Proc. R. Soc. A373, 27 (1980); A388 (1983).

[16] P.G. Kusalik, J. Chem. Phys. 93, 3520 (1990).

[17] For the present dense strongly dipolar systems the dielectric constants are sufficiently large that the reaction field is essentially independent of the exact value 15 . Therefore, conducting boundary conditions are an appropriate choice.

[18] The critical volume fractions are 0.157 for the Ising system and 0.295 for the XYZ model [1]. Using the effective hard sphere diameter $0.967 \sigma$ [see J.P. Hansen and I.R. McDonald, Theory of Simple Liquids, 2nd ed. (Academic, London, 1986), chap. 6] the soft-sphere densities $\rho^{*}=0.8$ and 1.05 correspond to volume fractions of 0.379 and 0.497 , respectively.

[19] The results given in Fig. 1 are for typical frozen configurations. Tests show that the actual values of $P$ vary a little from configuration to configuration but that the same general trend is found. Ideally, accurate values of $P$ could be obtained by averaging over many frozen configurations for each $N$. However, this would be very expensive computationally and is not necessary here; we are only interested in the limiting value of $P$ as $N$ becomes large and not in its detailed $N$ dependence.

[20] M.J.P. Gingras, Phys. Rev. Lett. 71, 1637 (1993).

[21] B. Bergersen and Z. Rácz, Phys. Rev. Lett. 67, 3047 (1991); H-J Xu, B. Bergersen, and Z. Rácz, Phys. Rev. E 47, 1520 (1993)
FIG. 1. The polarization $P$ at $T_{\min }^{*}$ vs $100 / N$ for the randomly frozen Ising (circles), XY (squares) and XYZ (triangles). Results are included for 108 (XYZ only), 256, 392 (XYZ only), 500 and 864 particles.

FIG. 2. $\quad P$ vs $T^{*}$ (rotational) for dynamically random $X Y Z$ systems. The squares, triangles and circles are for $m^{*}=1,5$ and 10, respectively. The error bars represent one estimated standard deviation. $g_{\text {Binder }}$ vs $T^{*}$ (rotational) is shown in the inset for $N=64$ (squares), 108 (triangles) and 256 (circles) particles.

FIG. 3. $\quad P$ vs $T^{*}$ (rotational) for the Ising model. Results are shown for dynamically random systems with $m^{*}=1$ (squares) and $m^{*}=5$ (triangles) and for the randomly frozen case (circles). The heat capacities per particle, $C_{v} / N$, are plotted vs $T^{*}$ (rotational) in the inset.

FIG. 4. The mass dependence of the disordered to ferroelectric transition temperature $T_{F}^{*}$ (rotational). The squares and triangles are results for the XY and XYZ models, respectively. The values of $T_{F}^{*}$ were obtained from plots of the heat capacity, $C_{v} / N$, vs $T^{*}$ (rotational) and a typical example (XYZ model, $m^{*}=1$ ) is shown in the inset. The error bars represent estimated uncertainties in the peak position. 